\title{
Editorial: Novel Therapies for Combating Bone Diseases Through Advances in Bone Remodeling
}

\author{
Yurui Jiao ${ }^{1}$, Jiake $X u^{2 *}$, Chao Liang $^{3,4 *}$ and Changjun $\mathrm{Li}^{1 *}$ \\ ${ }^{1}$ Department of Endocrinology, Endocrinology Research Center, Xiangya Hospital, Central South University, Changsha, \\ China, ${ }^{2}$ School of Biomedical Sciences, The University of Western Australia, Perth, WA, Australia, ${ }^{3}$ School of Chinese \\ Medicine, Law Sau Fai Institute for Advancing Translational Medicine in Bone and Joint Diseases, Hong Kong Baptist \\ University, Hong Kong, Hong Kong, SAR China, ${ }^{4}$ Department of Biology, Southern University of Science and Technology, \\ Shenzhen, China
}

Keywords: bone diseases, bone remodeling, bone cells, molecular targets, translational medicine

\section{Editorial on the Research Topic}

\section{Novel Therapies for Combating Bone Diseases Through Advances in Bone Remodeling}

OPEN ACCESS

Edited and reviewed by: Ramani Ramchandran Medical College of Wisconsin, United States

*Correspondence: Changjun Li lichangjun@csu.edu.cn Jiake $X u$

jiake.xu@uwa.edu.au

Chao Liang

liangchao512@163.com

Specialty section

This article was submitted to Molecular and Cellular Pathology,

a section of the journal Frontiers in Cell and Developmental

Biology

Received: 30 August 2021 Accepted: 21 September 2021 Published: 14 October 2021

Citation:

Jiao Y, XU J, Liang C and Li C (2021) Editorial: Novel Therapies for Combating Bone Diseases Through Advances in Bone Remodeling. Front. Cell Dev. Biol. 9:766963. doi: 10.3389/fcell.2021.766963
Bone is a metabolically active organ that undergoes a constant and continuous state of remodeling throughout life, which is important for the maintenance of normal skeletal structure and function (Salhotra et al., 2020). The coordinated action between bone cells, which are composed of osteoblasts, osteoclasts, and osteocytes, is known as bone remodeling, and the imbalance between the functioning of these cells leads to bone diseases such as osteoporosis, osteoarthritis, rheumatoid arthritis, and bone tumors (Kular et al., 2012; Chen et al.). Bone marrow mesenchymal stem cells (BMSCs), bone-derived exosomes, and microRNAs (miRNAs), which are involved in the regulation of skeletal metabolism, bone remodeling, and bone diseases, have been the current focus of research in the world (Mori et al., 2019; Kalluri and LeBleu, 2020). In recent years, an increasing number of studies were enriched in the area of bone remodeling by researchers, and the most results were encouraging (Li et al., 2015, 2018). These developments, which will provide theoretical advances on bone remodeling and have a growing impact on the treatment of bone diseases in the coming years.

This editorial paper collects 9 publications aimed to explore recent developments with a focus on identifying molecular mechanisms and targets in bone remodeling as well as new therapies for bone disease and associated complications.

\section{BONE MARROW MESENCHYMAL STEM CELLS}

BMSCs display increased adipogenic with age, along with decreased osteogenic differentiation capacity. Based on this theory, one study, Peng et al. identified a differentially expressed ASPH gene, which regulates Wnt signaling mediated by Gsk $3 \beta$, in middle-aged and elderly aged groups. The depletion of mouse Asph suppressed the capacity of osteogenic differentiation and accelerated cellular senescence in BMSCs, while the overexpression of Asph enhanced the capacity of osteogenic differentiation and inhibited cellular senescence. Because of the abundant expression of ASPH in a variety of malignant tumors, ASPH has been thought to be a potential therapeutic target for different cancers. Thus, they suggested that the treatment of ASPH inhibitor in patients with cancers needs to be concerned because of their potential risks of bone loss or bone fracture. 
In a second study, Li et al. explored the effect of calcitonin gene-related peptide (CGRP) on the osteogenic and adipogenic differentiation potential of BMSC. In this study, they indicated that CGRP promoted the osteogenic differentiation of BMSCs while inhibiting their adipogenic differentiation. Aged and ovariectomized mice treated with CGRP showed a substantial promotion of bone formation and a reduction in fat accumulation in the bone marrow. In another study, Xie et al. summarized the significance of Yes-associated protein 1 (YAP1) in orthopedic degenerative diseases. YAP1 can regulate the osteogenic differentiation of BMSCs, as well as the activity of osteoblasts and osteoclastogenesis. Therefore, the regulation of ASPH, CGRP, and YAP1 activity is expected to become a potential intervention strategy to delay the occurrence and development of skeletal degenerative diseases.

\section{BONE-DERIVED EXOSOMES AND MIRNAS}

Emerging numbers of studies have reported miRNAs as important regulators for bone metabolism. Yin et al. focused on the function and mechanism of miR-129-5p in bone metabolism recently and found that the expression of miR-129-5p was enhanced in both aging and menopause osteoporosis models. Overexpression and down-regulation of miR-129-5p respectively inhibited or enhanced osteoblasts differentiation and bone formation by regulating downstream transcription factors of the $\mathrm{Wnt} / \beta$-catenin pathway through targeting Tcf4. Moreover, bioengineered novel recombinant miR-129-5p inhibitor showed a rescue effect on osteoporosis.

Bone-derived exosomes are involved in the regulation of skeletal metabolism, bone remodeling, and pathological processes through modulating intercellular communication and the transfer of materials (Zhu et al., 2018). The founding of $\mathrm{Lu}$ et al. suggested that BMSC-derived exosomal miR29a regulates angiogenesis and osteogenesis, and miR-29aloaded BMSCs-Exosomes may serve as a robust and potential therapeutic target for osteoporosis. Besides, Lyu et al. and Liu et al. reviewed the current knowledge of exosomes and highlight the application studies of bone-derived exosomes in bone remolding and bone disorders. They summarized the role of exosomes derived from BMSCs, osteoclasts, osteoblasts, and osteocytes in skeletal metabolism, including miR-27a, miR-206a, miR-196a, miR-214, miR-30d-5p, miR133b-3p, miR-140-3p, miR-140-5p, miR-335-3p, miR-378b, miR218, miR-1192, miR-680, miR-302a, miR-92a-3b, miR-135b, and miR-100-5p.

\section{OSTEOCLASTS}

The interaction of receptor activator of nuclear factor-kB ligand (RANKL) and its receptor RANK is one of the fundamentals in bone remodeling. The RANK/RANKL system is well-known for regulating bone turnover by promoting the differentiation and activation of osteoclasts and has been shown to be a novel effective therapeutic target for osteoporosis. Zhang et al. focused on and summarized the advantages and disadvantages of the use of denosumab, the anti-RANKL antibody, in the treatment of postmenopausal osteoporosis. Although denosumab decreases osteoclast-mediated bone resorption and turnover, adverse events have also been reported after treatment, including skin eczema, flatulence, cellulitis, and osteonecrosis of the jaw. Considering the potential side effects of long-term medication of denosumab, aptamer has shown advantages and low toxicity and was hypothesized to be a promising candidate for therapeutic drugs targeting RANKL to counteract osteoporosis. Therefore, the determination of the three-dimensional structure of the RANKL-aptamer complex is necessary to discover the accurate binding domains and could be a crucial research basis for a functional aptamer targeting RANKL for the treatment of osteoporosis.

Protein kinase $\mathrm{C}$ delta $(\mathrm{PKC}-\delta)$ functions as an important regulator in bone metabolism. Rong et al. conducted an osteoclast-specific PKC- $\delta$ knockout mouse strain to explore the function of PKC- $\delta$ in osteoclast biology. They found that ablation of PKC- $\delta$ in osteoclasts resulted in an increased bone volume in male mice, accompanied by decreased Cathepsin-K protein levels, osteoclast number, osteoclast formation, and resorption, whereas these changes were not observed in female mice. The work of this study revealed a previously unknown target for the treatment of gender-related bone diseases. In these two studies, they summarized the significance of RANKL-aptamer and PKC$\delta$ and discuss the potential therapeutic strategies of the targeted modulation of the RANK/RANKL system and PKC- $\delta$ for bonerelated diseases.

Taken together, increasing regulators have been revealed to participate in bone remodeling, which facilitates the mechanistic understanding of bone diseases and associated complications caused by disruption of bone remodeling, and provides new strategies for the treatment of bone diseases.

\section{AUTHOR CONTRIBUTIONS}

All authors listed have made a substantial, direct and intellectual contribution to the work, and approved it for publication.

\section{FUNDING}

This work was supported by National Key R\&D Program of China (Grant No. 2019YFA0111900), the National Natural Science Foundation of China (Grant Nos. 81922017, 81873669, 81802209, 81930022, 91749105, 81520108008, and 81873670), Hunan Provincial Science and Technology Department (2018RS3030), and the Australian National Health and Medical Research Council (NHMRC, APP1107828, APP1127156, and APP1163933).

\section{ACKNOWLEDGMENTS}

We thank the authors of the papers published in this Research Topic for their valuable contributions. 


\section{REFERENCES}

Kalluri, R., and LeBleu, V. S. (2020). The biology, function, and biomedical applications of exosomes. Science. 367. doi: 10.1126/science.aau6977

Kular, J., Tickner, J., Chim, S. M., and Xu, J. (2012). An overview of the regulation of bone remodelling at the cellular level. Clin. Biochem. 45, 863-873. doi: 10.1016/j.clinbiochem.2012.03.021

Li, C. J., Cheng, P., Liang, M. K., Chen, Y. S., Lu, Q., Wang, J. Y., et al. (2015). MicroRNA-188 regulates age-related switch between osteoblast and adipocyte differentiation. J. Clin. Invest. 125, 1509-1522. doi: 10.1172/JCI7 7716

Li, C. J., Xiao, Y., Yang, M., Su, T., Sun, X., Guo, Q., et al. (2018). Long noncoding RNA Bmncr regulates mesenchymal stem cell fate during skeletal aging. J. Clin. Invest. 128, 5251-5266. doi: 10.1172/JCI9 9044

Mori, M. A., Ludwig, R. G., Garcia-Martin, R., Brandão, B. B., and Kahn, C. R. (2019). Extracellular miRNAs: From Biomarkers to Mediators of Physiology and Disease. Cell Metab. 30, 656-673. doi: 10.1016/j.cmet.2019. 07.011

Salhotra, A., Shah, H. N., Levi, B., and Longaker, M. T. (2020). Mechanisms of bone development and repair. Nat. Rev. Mol. Cell Biol. 21, 696-711. doi: 10.1038/s41580-020-00279-w
Zhu, S., Yao, F., Qiu, H., Zhang, G., Xu, H., and Xu, J. (2018). Coupling factors and exosomal packaging microRNAs involved in the regulation of bone remodelling. Biol. Rev. Camb. Philos. Soc. 93, 469-480. doi: 10.1111/brv.1 2353

Conflict of Interest: The authors declare that the research was conducted in the absence of any commercial or financial relationships that could be construed as a potential conflict of interest.

Publisher's Note: All claims expressed in this article are solely those of the authors and do not necessarily represent those of their affiliated organizations, or those of the publisher, the editors and the reviewers. Any product that may be evaluated in this article, or claim that may be made by its manufacturer, is not guaranteed or endorsed by the publisher.

Copyright (c) 2021 Jiao, Xu, Liang and Li. This is an open-access article distributed under the terms of the Creative Commons Attribution License (CC BY). The use, distribution or reproduction in other forums is permitted, provided the original author(s) and the copyright owner(s) are credited and that the original publication in this journal is cited, in accordance with accepted academic practice. No use, distribution or reproduction is permitted which does not comply with these terms. 